\title{
ACTITUDES HACIA EL APRENDIZAJE DE LAS DANZAS FOLCLÓRICAS EN ESTUDIANTES DE SEXTO DE PRIMARIA
}

\author{
Rodolfo Eloy Tolentino Escarcena* \\ Universidad Católica Sede Sapientiae \\ rodolfo.tolentino.1921@gmail.com
}

\author{
Aldo Medina Gamero** \\ Universidad Católica Sedes Sapientiae \\ medrafa222@gmail.com
}

\author{
Carlos Luy-Montejo ${ }^{* * *}$ \\ Universidad Católica Sedes Sapientiae \\ fracarlitos@gmail.com
}

* Rodolfo Eloy Tolentino Escarcena es doctorando en Ciencias de la Educación y magíster en Docencia e Investigación Superior. ORCID: 0000-0003-2480-5869.

" Aldo Medina Gamero es doctorando en Humanidades, magíster en educación e investigador Renacyt.

"*. Carlos Luy-Montejo es doctor en Educación y Docencia Universitaria. Es también magister en Educación e Investigación, así como investigador Renacyt. ORCID ID: 0000-0003-0824-7959. 
Fecha de recepción: agosto de 2020

Fecha de aceptación: diciembre de 2020

Resumen: El trabajo de investigación pertenece al campo de las ciencias sociales, específicamente al abordaje de las danzas folklóricas peruanas como parte del aprendizaje dentro del área de Arte y Cultura en la Educación Básica Regular. Por ello, la unidad de análisis está conformada por los alumnos de sexto grado de una institución educativa estatal en Pachacamac. El objetivo fue medir las características de las actitudes hacia la danza folklórica considerando que esta investigación descriptiva puede diagnosticar los niveles de actitudes y constituir un primer estadio para otros niveles de investigación. Los objetivos específicos fueron propuestos por cada dimensión (cognitivo, conductual y afectivo) basado en la teoría de las actitudes propuestas por Morales (1999). Las actitudes son estados internos no observados de manera directa. Por ello, es necesario buscar los medios para determinar la orientación del sujeto hacia determinado hecho. La muestra fue de tipo no probabilista por conveniencia, ya que los estudiantes fueron formados de acuerdo con el alcance que se tuvo por cuestiones de acceso. El tipo de investigación es cuantitativa - transversal de nivel descriptivo. La recolección de los datos fue realizada mediante una escala tipo Likert denominada Escala de actitudes hacia las danzas folclóricas. Consta de cuatro valores "nunca", "casi nunca", "siempre" y "casi siempre". Para las validaciones se realizó la validez por juicio de expertos junto a la prueba V de Aiken; además de la fiabilidad a través del coeficiente de consistencia interna de alfa de Cronbach. El estudio concluye que los alumnos de una institución educativa estatal en Pachacamac tienen, en su mayoría, un nivel alto de actitudes hacia las danzas folklóricas.

Palabras claves: Actitudes, educación, danza, folklore, aprendizaje. 


\title{
ATTITUDES TOWARDS LEARNING FOLK DANCES IN SIXTH ELEMENTARY STUDENTS
}

\begin{abstract}
The research work belongs to the field of social sciences, specifically to the approach of Peruvian folk dances, these as part of learning within the area of Art and Culture in Regular Basic Education. Therefore, the unit of analysis is made up of sixth grade students from a state educational institution in Pachacamac. The objective was to measure the characteristics of attitudes towards folk dance considering that this descriptive research can diagnose the levels of attitudes and constitute a first stage for other levels of research. The specific objectives were proposed for each dimension (cognitive, behavioral and affective) based on the theory of attitudes proposed by Morales (1999). Attitudes are internal states not directly observed. Therefore, it is necessary to find a mechanism to determine the orientation of the subject towards a certain fact. The sample was nonprobabilistic for convenience; since, the students were trained according to the scope that was had for access issues. The type of research is quantitative - transversal at the descriptive level. Data collection was carried out using a Likert-type scale called Scale of attitudes towards folk dances. It consists of four values "never", "almost never", "always" and "almost always". For the validations, the validity was performed by expert judgment together with the Aiken $\mathrm{V}$ test; in addition to the reliability through the internal consistency coefficient of Cronbach's alpha. The study concludes that the students of a state educational institution in Pachacamac have, for the most


part, a high level of attitudes towards folk dances.

Keywords: Attitudes, education, dance, folklore, learning.

\section{Introducción}

iertamente, el aprendizaje en el ser humano constituye uno de los
mayores retos de la educación. Esto motivado por la búsqueda
en comprender cuál es la manera adecuada de lograr aprendizajes sustantivos que puedan verse expresados en la resolución de conflictos o la producción de instrumentos en determinados contextos (Gardner, 1999). Esa incesante exploración nos lleva a repensar en la investigación de aspectos más íntimos del sujeto vinculados a las actitudes que sirvan como hallazgos primigenios para que, al tratarlos, contribuyan en desarrollar procesos que mejoren su estado en el desarrollo de capacidades y competencias. A propósito, desde el Ministerio de Educación del Perú (Minedu, 2016) se proponen lineamientos para desarrollarlas; sin embargo, no se profundiza en un tratamiento - diagnóstico que inicie un círculo efectivo de aprendizaje.

Es evidente que en la educación peruana existen diversas áreas de estudio que han sido y son las que sugieren una mayor atención en el desarrollo del sistema educativo; no obstante, en la mayoría de casos, las de Arte, Arte y Cultura o Educación Artística, como quiera que se le denomine en los diversos modelos de currículo, no son tomadas en cuenta por su "bajo valor académico" y mayor estima en lo estético. Esta perspectiva carece de sentido, más aún, cuando se vincula al aprendizaje de la danza folklórica como uno de los lenguajes artísticos de esta área (Barzola, 2019).

Si se toma atención en que el aprendizaje del arte, en sus diversos lenguajes, no es un fenómeno apartado de toda la estructura educacional; 
se podrá entender que "su complejidad es enorme y, su posibilidad de desarrollo y avance exige estudios profundos y de mayor amplitud" (Palacios, 2006, p.19). Por tanto, centrar su atención en procesos que posibiliten su desarrollo a mediano plazo, podrá contribuir en el engranaje de todas las competencias que debe desarrollar el ser humano para su formación como persona (Ministerio de Educación, 2016).

Ahora bien, la danza folklórica es uno de los lenguajes artísticos que es enseñado en las escuelas en todos los niveles, tipos o formas de educación. En tal sentido, ha permitido el desarrollo de una de las maneras de preservar la continuidad en la formación de la identidad cultural y nacional. Además, su práctica, implica el desarrollo de habilidades psicofísicas aun de terapia emocional.

En la misma línea, es importante, para este estudio, conocer las actitudes que emergen de las experiencias hacia las danzas folklóricas que tienen los alumnos de sexto grado durante su tránsito por la educación primaria en la Educación Básica Regular (EBR). De esta forma, su descripción y medición sirven de diagnóstico a los docentes para que promuevan estrategias adecuadas que favorezcan la mayor predisposición hacia su aprendizaje e incrementen sus conocimientos en los niveles cognitivo, afectivo y conductual.

\section{Marco de referencia}

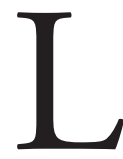

as actitudes tienen un amplio desarrollo teórico de conceptualización avalado por diversos enfoques y disciplinas de abordaje. Lo cierto es que todas estas atienden a considerar los polos positivo o negativo de tendencia hacia algún objeto de estudio en particular. Por ello, un breve recuento de conceptualización pertinente para el estudio nos va a permitir atender de modo preciso al objetivo de la investigación. 
En primera instancia, existe vinculación entre la actividad de pensar y actuar provocada por una necesidad motivacional (Pro, 2003). Esta necesidad traducida en interés va a determinar la tendencia y a la vez el grado de aceptación o rechazo hacia ciertos fenómenos. En la misma línea Pozo y Gómez (2011) reafirman esta tendencia al establecerlos como patrones anteriores al juicio para determinar vínculo con el objeto y que esto determine su conducta. En síntesis, la motivación tiene un rol importante en la toma de decisiones hacia determinado fenómeno u objeto; la que puede ser positiva y negativa sin la necesidad de existencia que lo vincule de manera apriorística. Sin embargo, Sarabia (1992) considera que el relativismo de nuestras decisiones condiciona cierto acercamiento con las características del objeto de estudio para demostrar cierta disposición.

Ahora bien, luego de sostener características relativamente comunes en la teoría de las actitudes, hemos sostenido los aportes de Morales (1999) como marco de referencia para el análisis de la investigación. Por ello, en consonancia con lo presentado, define a las actitudes como estados internos no observados de manera directa en donde coexisten “...tres tipos de respuestas como vías de expresión de un único estado interno (la actitud), explica la complejidad de dicho estado y también que muchos autores hablen de los tres componentes o elementos de la actitud" (p.195). 
Tabla 1

Componentes de las actitudes

Componentes de las actitudes

Componente cognitivo Componente afectivo Componente

conductual

Se sostiene en la valora- Componente vinculado Muestra de ma-

ción que tiene el sujeto con el estado de ánimo y las nera observable hacia determinada per- emociones. El sujeto evalúa su postura a favor sona, objeto o hecho sus preferencias de acuerdo o en contra hacia en función del conoci- con el carácter interno de la persona, objemiento que tiene sobre sus respuestas producto de to o hecho. Este el mismo. su experiencia. componente es el más relacionado con la actitud.

Nota. Adaptado de Psicología social, por Morales, 1999, p. 42.

Las actitudes sostenidas como la interacción entre estos tres componentes requieren de ciertos requisitos para su formación en el sujeto. De acuerdo con Oskamp (1999), es posible esta realización atendiendo a los siguientes factores:

- La experiencia personal directa que afecta directamente la relación entre el sujeto y el objeto de estudio. Ciertamente, la interacción entre ambos dentro de cierto intervalo de tiempo puede condicionar cierta tendencia a partir del tipo de relación recibida. 
- La influencia de los padres, como parte de los modelos de desarrollo de la persona y gran influencia en las primeras etapas de la vida, puede provocar una especie de enseñanza por imitación que se ve alimentado por la convivencia en el entorno familiar.

- La influencia del grupo o la presión que ejercida por esta a través de diversos mecanismos de socialización; disponen la influencia sustentada en la cantidad de tiempo al contacto entre pares sobre todo entre niños y adolescentes quienes se encuentran en proceso de formación de su carácter que se encuentra vinculado a la actitud.

- De la misma forma, los medios de comunicación de masas, a través de su gran capacidad de difusión, condicionan posturas a favor o en contra de determinados eventos.

Por tanto, el aprendizaje de las actitudes, de acuerdo con Guitart (2002), ocurre en la no contradicción entre los contextos que potencian su influencia. Mientras el sujeto no modifique constantemente sus esquemas cognitivos, conductuales o emocionales está propenso a arraigar ciertas actitudes. Pero este proceso puede ir modificándose, es decir, la actitud favorable o desfavorable, producto del tiempo, el ambiente, el entorno, las relaciones sociales, etc. Existen algunos elementos fundamentales involucrados al aprendizaje. Estos son los relacionados con los factores internos, la influencia de los agentes externos (otras personas) y el llamado objeto de la actitud.

Claramente, el enfoque predominante en la formación académica de las escuelas públicas es el constructivismo. Se basa en la relación entre los estados internos y el contexto que es lo que determina un nuevo aprendizaje haciéndolo significativo. Entonces, las actitudes se forman 
producto de la prolífica y variada interacción entre pares, el trabajo en equipo y/ colaborativo, la coevaluación, etc. Además, las sesiones de clase están estructuradas de manera que este punto sea el impulso para generar los aprendizajes. La actitud frente al área se erige entonces como el punto neurálgico dentro de las clases. En ese sentido, Moreno (2011) advierte que los buenos hábitos, las normas, los valores y los patrones de conducta dentro de una institución educativa se relacionan positivamente con el desarrollo de la competencia pedagógica del educando.

\section{Método}

T a investigación se ha desarrollado siguiendo el enfoque cuantitativo; debido a que la recolección de información ha sido procesada con el objetivo de obtener mediciones en función de las actitudes en estudiantes de sexto grado. Bajo el diseño descriptivo se procederá a caracterizar al fenómeno de estudio. Los participantes han sido conformados bajo el muestreo de tipo no probabilístico por conveniencia, tal como se detallará de la siguiente forma en la Tabla 2. 
Tabla 2

Caracterización de la muestra de estudio

Muestra

Edad

Grado

Nivel

Turno

Idioma

Nacionalidad

Distrito

\section{6 alumnos}

$11-12$ años

Sexto

Primaria

Mañana

Español (100\%)

Peruana $(100 \%)$

Pachacamac

El instrumento utilizado ha sido una escala de actitudes hacia las danzas folklóricas que ha seguido el criterio de validación por ocho jueces expertos bajo el coeficiente de $\mathrm{V}$ de Aiken con un valor de 0,72. Además, para la confiabilidad, el instrumento obtuvo un valor de 0.92 para el promedio de los 24 ítems sometidos al tratamiento estadístico del coeficiente de Alfa de Cronbach. Estos se encuentran agrupados en tres dimensiones, de acuerdo con la teoría propuesta, bajo una escala dicotómica $(0$ = en desacierto; 1 = acierto). En el contexto de este estudio se construyó una escala tipo Likert, que incluye las tres dimensiones de las actitudes (Morales, 1999): cognitiva (ítems 1,2,3,4,5,6,7,8), conductual (ítems 
$9,10,11,12,13,14,15,16)$ y afectivo (ítems 17,18,19,20,21,22,23,24). La escala está considerada como uno de los métodos más adecuados para medir actitudes ya que su estructura facilita la aplicación y calificación. Consiste en un conjunto de ítems en forma de afirmaciones o juicios, ante los cuales se pide a los participantes que exterioricen su reacción y elijan uno de los puntos de la escala (Hernández, Fernández \& Baptista, 2003).

\section{Resultados y conclusiones}

T a presentación de los resultados responde al análisis estadístico descriptivo sobre la variable de estudio y sus tres dimensiones.

Tabla 3

Estadisticos descriptivos para la variable actitudes hacia el aprendizaje de las danzas folklóricas

\begin{tabular}{lcccc}
\hline Variable & Media & Mediana & Desviación E. & Varianza \\
\hline Actitudes hacia & 70.8 & 71 & 9.30 & 86.58 \\
el aprendizaje & & & & \\
de las danzas & & & & \\
folklóricas & & & & \\
$\mathrm{N}=36$ & & & &
\end{tabular}

Los resultados presentan una media de 70.8 que se encuentra cerca del nivel absoluto de puntajes para un cuestionario de veinticuatro ítems con un valor de cuatro como escala más alta. Se precisa que la tendencia de las puntuaciones es unidireccional o en un solo sentido. 


\section{Tabla 4}

Estadisticos para las dimensiones de las actitudes hacia el aprendizaje de las danzas folklóricas

\begin{tabular}{lllll}
\hline Variable & Media & Mediana & Desviación E. & Varianza \\
\hline Dimensión cognitiva & 25.2 & 25.5 & 3.42 & 11.70 \\
Dimensión conduc- & 21.3 & 21 & 3,71 & 13,76 \\
tual & & & & \\
Dimensión afectiva & 24,3 & 25 & 3,90 & 13,76 \\
\hline $\mathrm{N}=36$ & & & &
\end{tabular}

De la misma forma, en las dimensiones sobre las actitudes hacia las danzas folklóricas se obtienen puntajes por encima del promedio. Esto evidencia una mayor predisposición en los tres componentes: cognitivo, conductual y afectivo.

Tabla 5

Niveles para la variable actitudes hacia el aprendizaje de las danzas folklóricas

\begin{tabular}{lcc}
\hline Niveles de la variable & Frecuencia & Porcentaje \\
\hline Nivel bajo & 0 & $0 \%$ \\
Nivel medio & 9 & $25 \%$ \\
Nivel alto & 27 & $75 \%$ \\
\hline
\end{tabular}

$\mathrm{N}=36$ 
Al realizar el análisis por niveles de medición de la variable de estudio, se obtienen que la mayoría de los estudiantes (tres cuartos del total) muestra actitud favorable hacia el aprendizaje de las danzas. Además, ninguno de los participantes muestra una tendencia negativa hacia esta.

De acuerdo con la teoría determinista en la que la conducta es determinada por la actitud (Escudero, 1998; Myers, 1995), se entiende que los estudiantes muestran actitudes positivas hacia el aprendizaje de las danzas folklóricas; tanto en su componente práctico como teórico. La sensibilidad expresada por una manifestación artística tiende a mostrar la subjetividad a través del lenguaje corporal.

Es interesante comprender que estas pruebas tomadas como preliminares pueden develar los efectos de aprendizajes previos (Morales et al, 2000). Es decir, la formación de las actitudes se presenta como consecuencia de la interrelación de las personas, el desarrollo de la cognición y el mismo aprendizaje.

Es preciso continuar con investigaciones al respecto para comprender las razones por las que las actitudes para este grupo de estudio son positivas e intentar homologar resultados con otras áreas del conocimiento.

\section{REFERENCIAS}

Escudero, T. (1975). Las actitudes en la enseñanza de las ciencias: un panorama complejo. Revista de educación, 5, 236241.

Gardner, H. (1999). Audiences for the theory of multiple intelligences. Basic Books. 
Guitar, R. (2002). Las actitudes en el centro escolar: Reflexiones y propuestas. Graó.

Ministerio de Educación. (2016). Programa Curricular de Eduación Básica Regular. Ministerio de Educación.

Morales, F.; Huici, C.; Moya, M.; Gaviria, E.; López, M. \& Encarnación, N. (2000). Psicología Social. Mc Graw Hill.

Morales, J. (1999). Psicología Social. Mc Graw Hill.

Moreno, A. (2011). La reproducción intergeneracional de las desigualdades educativas: límites y oportunidades de la democracia. Revista de educación, 83-208. https://sede.educacion.gob.es/ publiventa/d/22948/19/0

Myers, D. (1995). Psicología Social. Mc Graw Hill.

Oskamp, S. (1999). Factors influencing household recycling behavior. Environment and Behavior, 23(4), 494-519. https://doi. org/10.1177/0013916591234005

Palacios, L. (2006). El valor del arte en el proceso educativo. Reencuentro, 46, 1-21. https://www.redalyc.org/articulo.oa?id=34004607

Pro, A. (2003). La construcción del conocimiento científico y los contenidos de ciencias. En M. P. Jiménez (coord.), Enseñar ciencias (pp. 33-54). Graó.

Pozo, I., \& Gómez, M. (2011). Aprender y enseñar ciencia. Morata.

Sarabia, B. (1992). El aprendizaje y la enseñanza de las actitudes. Santillana. 